\title{
Experimental Validation of a SDN Orchestrator for the Automatic Provisioning of Fixed and Mobile Services
}

\author{
Ricardo Martínez ${ }^{(1)}$, Ricard Vilalta ${ }^{(1)}$, Arturo Mayoral ${ }^{(1)}$, Ramon Casellas ${ }^{(1)}$ and Raül Muñoz ${ }^{(1)}$ \\ (1) CTTC, Av. Carl Friedrich Gauss 7, Castelldefels, 08860, Spain, rmartinez@cttc.es
}

\begin{abstract}
We design, implement and experimentally validate an SDN orchestrator for the automatic provisioning and seamless transport of fixed and mobile services over a multi-layer aggregation network. The proposed solution aims at fostering fixed mobile convergence solutions for future $5 G$ networks.
\end{abstract}

\section{Introduction}

Bandwidth usage at both fixed and mobile access networks is continuously growing and this trend will continue in coming years. Despite this, the revenues for service operators do not increase proportionally ${ }^{1}$. Aiming at keeping the competiveness, operators are seeking for scalable, energy-efficient and optimized network solutions that besides coping with the increasing traffic do minimize both CapEx and OpEx. To this end, an appealing solution fosters the fixed mobile convergence (FMC) concept which is seen as crucial for future $5 \mathrm{G}$ networks ${ }^{2}$.

Traditionally FMC is attained at the service level (IP and IP Multimedia Subsystems) where fixed and mobile networks were designed and evolve independently. However, a more effective and efficient FMC strategy aims are deploying solutions at the network level from a twofold perspective ${ }^{2}$ : structural and functional. The former focuses on sharing and unifying equipment/technologies to transport both fixed and mobile traffic flows over the same network. The later, functional convergence, explores common entities and functions (e.g., unified control and management, AAA operations, etc.) that are applied regardless of the service type.

Herein both structural and functional convergence are achieved over a multi-layer (packet and optical) aggregation network controlled by a unified Software Defined Network (SDN) orchestration system. The multilayer aggregation network (structural convergence) leverages the benefits of both packet (i.e., granularity, statistical multiplexing) and optical (huge capacity) switching to seamlessly transport QoS-enabled packet flows ${ }^{1}$. Hence, multiple mobile and/or fixed services are grouped and carried over the same infrastructure ${ }^{3}$. The automatic provisioning of such flows is handled by the SDN orchestration. The considered SDN orchestrator follows the architecture defined on the Application-Based Network Operations (ABNO) ${ }^{3}$. This provides achieving the targeted functional convergence.
We present the implemented ABNO orchestrator wherein both fixed and mobile client applications running on top of it automatically instantiate connections within the aggregation network. Then, the experimental validation is carried out though the setting up of mobile LTE services (Evolve Packet System -EPS- Bearers) between cell stations (eNBs) and the Evolved Packet Core (EPC), responsible for the Internet access for the mobile users. The validation is shown from a twofold perspective: i) at the control plane level, it is detailed the ABNO building blocks interactions and exchanged heterogeneous control messages, including OpenFlow extensions; ii) at the data plane level, the traffic flow adaptation to actually transport EPS Bearers over the multi-layer (MPLS and optical) network infrastructure.

\section{Architecture of the ABNO for functional FMC}

The considered SDN-based ABNO orchestrator is depicted in Fig.1. The Service Controller handles incoming service requests from both fixed and mobile service applications. These applications trigger the service demands (via REST API) specifying the type of transport service (e.g., MPLS), endpoints (e.g., for mobile connections the eNBs and EPC Serving Gateway - SGW), the requested bandwidth and other QoS requirements (e.g., maximum latency). The PCE computes a multi-layer path including both packet and optical infrastructure. The objective function besides dealing with the QoS requirements is to attain the most efficient use of network resources at both layers fostering the packet grooming into established optical circuits ${ }^{5}$. The topology server is a database where full (or partial/abstracted) network connectivity and resource attributes at both layers are gathered. This is the input for the PCE computations. The provisioning manager function coordinates the corresponding SDN controllers to actually conduct the network elements configuration along the computed paths. As shown in the Fig.1, in the aggregation 
network two heterogeneous control plane solutions (one per switching technology) are adopted: the control of MPLS switches is done by an SDN packet controller (Ryu); and the control of optical circuits is handled by an Active Stateful (AS) PCE. The former relies on the OpenFlow 1.3 protocol to configure the forwarding of the MPLS nodes (i.e., MPLS tag pop/push). The latter enables instantiating optical connections via a distributed GMPLS control plane. Last but not least, the Flow Server keeps the active packet flows.

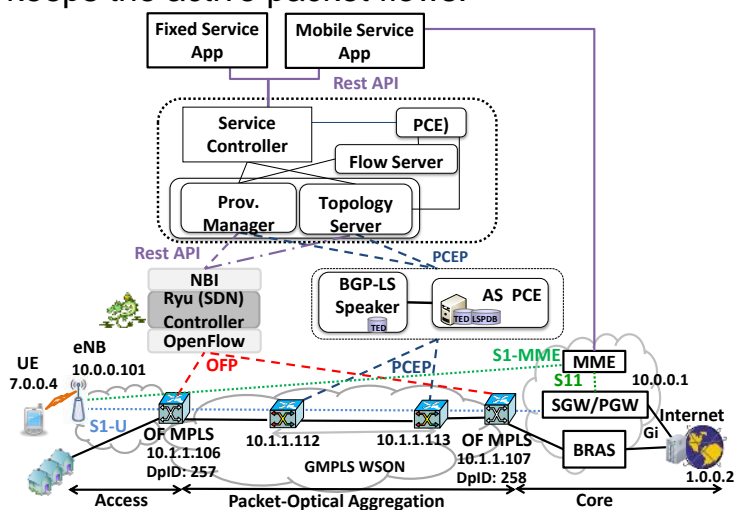

Fig. 1: Multi-layer aggregation ABNO orchestrator for FMC.

The logical interfaces/APIs enabling the communication among the aforementioned ABNO elements (including SDN Ryu controller and AS PCE) relies on combining both REST and PCEP interfaces. For instance, when a new MPLS flow transporting an EPS Bearer needs to be set up, a multi-layer path is computed by the PCE. The computed path is passed to the provisioning manager which coordinates both the SDN Ryu controller and the AS PCE to perform the transport network configuration. That is, it allows setting up the MPLS flow associated to the EPS Bearer and (if needed) the optical tunnel accommodating such packet flow. Observe that new MPLS flows can be tunnelled over existing optical tunnels as long as sufficient available bandwidth on those optical circuits exist (i.e., for grooming purposes).

\section{Experimental Validation}

The experimental setup is shown in Fig. 1. It is basically formed by three main elements: the LTE-EPC network provided by the LENA emulator $^{3}$ (including User Equipment and eNBs), the multi-layer OF-enabled MPLS ${ }^{7}$ - optical aggregation network deployed within the ADRENALINE testbed ${ }^{5}$ and the unified ABNO orchestrator discussed above. Without losing of generality, the experimental validation aims at transporting mobile services (EPS Bearers) between the eNBs and the EPC (SGW) via the multi-layer aggregation infrastructure. All the mechanisms and functions to do so are automatically coordinated by the ABNO orchestrator. For the sake of completeness, the ABNO operates similarly when fixed services need to be set up.

Focusing on the mobile services, once the EPS Bearer is negotiated between the eNB and the EPC Mobile Management Entity (MME) ${ }^{6}$ through the (out-of-band) control S1-MME interface, the MME communicates with the Mobile Service App (running on top the ABNO) to request the transport of EPS Bearer data/user packets (i.e., S1-U interface ${ }^{6}$ ). The interface between the MME and Mobile Service Apps is implemented using a REST API. Indeed, a new generic service call is defined to request from both Mobile and Fixed Service Apps, multi-layer transport connections handled by the ABNO.

For the EPS Bearers, the service call specifies the endpoint IP addresses (i.e., eNB and SGW), the requested bandwidth (ReqBw) in $\mathrm{Gb} / \mathrm{s}$, and specific match attributes (such as mobile data packet attributes). The latter allows mapping EPS Bearers with specific MPLS flows within the aggregation network. Specifically, EPS Bearers use the GPRS Tunneling Protocol (GTP-U) to transport data packets between the eNB and the EPC. Each EPS Bearer flow (S1$U$ ) has an individual Tunnel Endpoint Identifiers (TEID) which is carried into the GTP-U protocol. In this work we bind such TEIDs with individual MPLS labels. That is, we apply a policy where every EPS Bearer (with its own TEIDs) is transported over a different MPLS flow. Nevertheless, multiple MPLS flows may be aggregated into a unique optical tunnel.

Fig. 2 a) depicts the flowchart and messages exchanged among the ABNO elements when a (mobile) service call is received. Such a message is encapsulated into a REST POST message (step 1 in Fig.2.a) specifying the transport service type (e.g., MPLS), the endpoints (eNB at 10.0.0.101; SGW 10.0.0.1), the TEIDs (set to 2) and the ReqBw. Upon receiving the service call request, the Service Controller first determines the ingress and egress MPLS switches connected to the source and destination nodes (i.e., eNB and SGW). Next, the Service Controller sends a PCE Request (PCReq) to the PCE (step 2) with the MPLS endpoints. The PCE computes the route between those MPLS nodes considering the ReqBw. To do so, the PCE must retrieve the network topology. This is achieved via a request to the topology server function (i.e., REST GET Packet Topology). The topology server then asynchronously queries to both the SDN controller (for packet infrastructure) and the AS PCE (for the optical network) to update their respective network status (step 3 in Fig.2.a). 
Once the network topology is updated, the PCE executes the path computation. If a feasible path succeeds, a PCE response (PCRep) with the computed path (i.e., Explicit Route Object, ERO) is returned to the Service Controller (step 4).

After the path is computed, the setting up of the multi-layer transport connection must be done sending to the provisioning manager a Packet Connection Establishment Request message (step 5). This message carries the computed ERO and, for the mobile service, the associated matching rules: TEIDs and source and destination IP addresses of the EPS Bearer. The provisioning manager communicates with both SDN packet controller and AS PCE to perform the configurations on both layers.

As mentioned above, the AS PCE is used when the establishment of a new optical connection is needed requiring the configuration of the optical transceiver (i.e., WDM channel) and the wavelength switching nodes.

On the other hand, in the SDN packet controller, the extended OpenFlow 1.3 OFPT_FLOW_MOD message with experimental matches configures the MPLS nodes according to the EPS Bearer attributes (S1-U interface). That is, for each EPS Bearer, the GTP-U packet is encapsulated and decapsulated over a MPLS tunnel. As shown in Fig.2.b, the OFPT_FLOW_MOD (Fig.2.b) carries a set of match rules and actions for the MPLS nodes that define the processing and treatment of the data packet (GTP-U) of each EPS Bearer. In the example, the match rules impose that: all the GTP-U packets received over an incoming port with the tuple formed by a determined pair of source and destination IP addresses (i.e., eNB 10.0.0.101 and SGW 10.0.0.1), UDP port set to 2152 and the TEID equals to 2 , then a MPLS tag (1002) is pushed and the resulting packet is

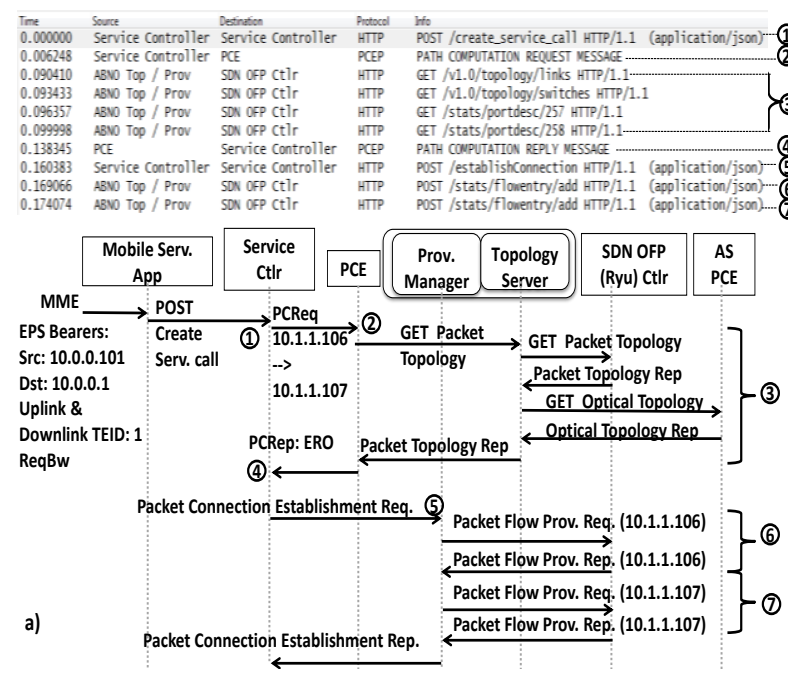

forwarded to the output port towards the optical domain. Similar operations but removing the MPLS tag are done when the EPS Bearer leaves the MPLS domain prior to be delivered to either eNB or SGW (downlink and uplink flows).

\section{Conclusions}

We have experimentally validated an SDN orchestration system based on the ABNO architecture to provide the automatic provisioning of both fixed and mobile services over a multi-layer (packet - optical) aggregation network. This allows attaining both functional and structural FMC objectives for future 5G networks where a single centralized control entity (ABNO) handles both types of services which are seamlessly transported over the common aggregation infrastructure.

\section{Acknowledgements}

This works was partially funded by the EC's FP7 (IP COMBO project - 317762) and by Spanish MINECO FARO project (TEC2012-38119).

\section{References}

[1] A. Mathew et al., "Multi-Layer High-Speed Network Design in Mobile Backhaul Using Robust Optimization", JOCN (2015)

[2] S. Gosselin et al., "Fixed and Mobile Convergence: Which Role for Optical Networks?" OFCTh3H.2, (2015).

[3] N. Baldo et al., "A Testbed for Fixed and Mobile Convergence Experimentation: ADRENALINE-LENA Integration", Proc. European Wireless (2014).

[4] A. Mayoral et al., "Experimental Seamless Virtual Machine Migration using an Integrated SDN, IT and Network Orchestrator" Proc OFC 2015.

[5] R. Martinez et al., "Experimental Evaluation of Delay-Sensitive Traffic Routing in Multi-Layer (Packet-Optical) Aggregation Networks for Fixed Mobile Convergence", ECOC (2013).

[6] 3GPP, "E-UTRA and E-UTRAN overall description", TS 36.300, March 2012.

[7] M. Suné et al., "An OpenFlow Implementation for Network Processors", Proc. EWSDN 2014

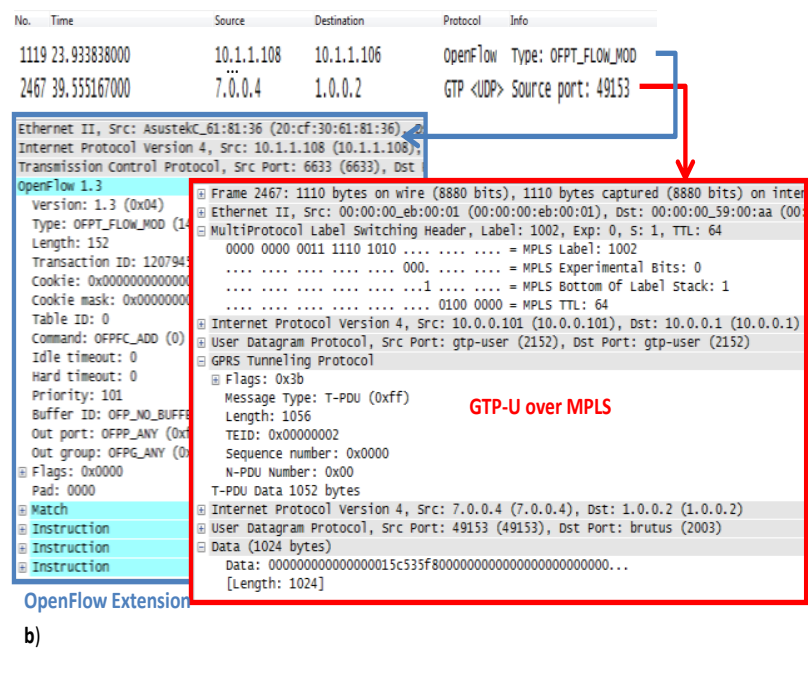

Fiq. 2: a) Creation of the mobile service via ABNO controller; b) OFP Extension and GTP-U over MPLS 